\title{
Impact of Antisymmetric Anisotropy on Magnetisation of Ultra-Hard Nanocrystalline Alloys
}

\author{
G. ZióŁKOWski* AND A. Chrobak \\ Institute of Physics, Silesian University, Uniwersytecka 4, 40-007 Katowice, Poland
}

\begin{abstract}
This paper describes an application of two-level Stoner-Wohlfarth model including antisymmetric anisotropy and two-level energetic model for simulations of magnetization processes in nanocrystalline, ultra-hard magnetic alloys. The simulation results have been compared with the experimental data obtained for the $\left(\mathrm{Fe}_{80} \mathrm{Nb}_{6} \mathrm{~B}_{14}\right)_{0.88} \mathrm{~Tb}_{0.12}$ alloy. Moreover, the correctness of the proposed model was confirmed by the quantitative agreement with the direct Monte Carlo simulations of similar magnetic systems.
\end{abstract}

DOI: 10.12693/APhysPolA.130.1137

PACS/topics: 75.50.Ww, 75.30.Gw, 75.50.Tt

\section{Introduction}

Magnetic materials are very important in nowadays technologies. New and continuously increasing requirements can be fulfilled by modern nanostructured magnetic composites containing different phases characterized by different magnetic properties. In the field of hard magnets, interactions between the phases are especially important and can lead to an appearing of new and unique properties [1,2]. Recently, we reported ultrahigh coercivity (about $3.6 \mathrm{~T}$ ) in $\mathrm{Fe}-\mathrm{Nb}-\mathrm{B}-\mathrm{Tb}$ type of bulk nanocrystalline alloys prepared by vacuum suction casting technique [3]. Figure 1 shows an example for the $\left(\mathrm{Fe}_{80} \mathrm{Nb}_{6} \mathrm{~B}_{14}\right)_{0.88} \mathrm{~Tb}_{0.12}$ nanocrystalline alloy. In this case interesting is the asymmetry of the hysteresis loop that suggests an occurrence of soft, hard and ultra-hard interacting magnetic phases. The ultra-hard phase does not directly contribute to magnetization process but via some interactions influences the remaining phases.

From the both, scientific as well as application point of view, it is a key challenge to understand the role of interactions with ultra-hard phases and consequently, magnetization processes in such materials.

In this work, we present models and simulations, based on the Stoner-Wohlfarth [4] and the two-level energetic model [5], of the magnetization process for high-coercive magnetic nanocomposites. In the investigated system, the magnetic objects with extremely high coercivity are "frozen" after the first magnetization and next, they cannot contribute to magnetization change, being a source of additional anisotropy, called exchange anisotropy. Interactions between the frozen objects and their surroundings leads to occurrence of some internal preferred magnetization directions. We propose a model in which the exchange anisotropy is simulated by antisymmetric uniaxial anisotropy constants originating from the ultra-hard magnetic objects and acting on the remaining part of the composite.

\footnotetext{
* corresponding author; e-mail: gziolkowski@us.edu.pl
}

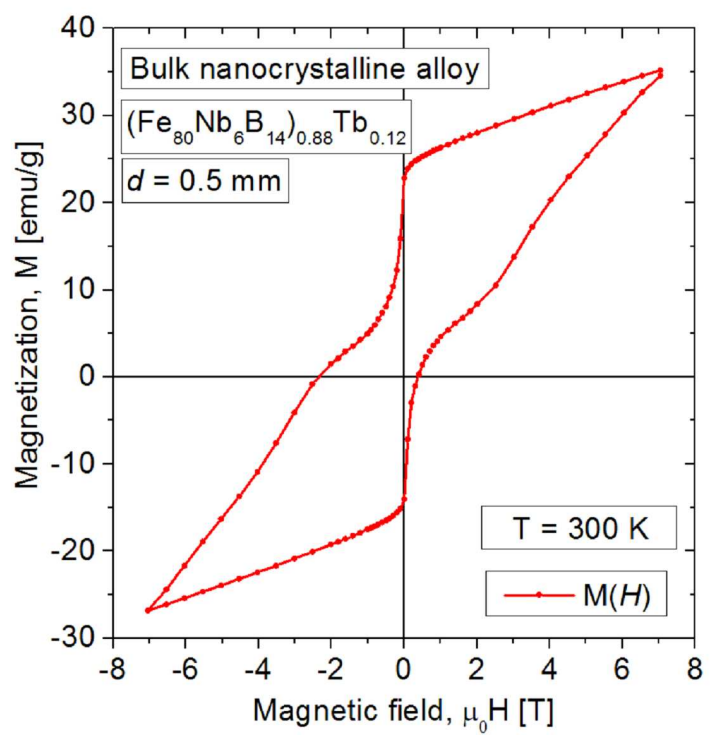

Fig. 1. Magnetic hysteresis loop for the $\left(\mathrm{Fe}_{80} \mathrm{Nb}_{6} \mathrm{~B}_{14}\right)_{0.88} \mathrm{~Tb}_{0.12}$ alloy [3].

\section{Model and simulation procedure}

The magnetic properties of the non-interacting, single domain nanoparticles (at temperature $T=0 \mathrm{~K}$ ) describes the so-called Stoner-Wohlfarth (S-W) model [4]. The key point of the model is the energy $E$ calculation of each magnetic object as a function of $\theta$ (angle between the applied field and the easy magnetization axis of the object) including magnetocrystalline anisotropy, shape anisotropy, and interaction with magnetic field. Figure 2a presents three examples of such function for different ratio of magnetic energy and anisotropy coefficient. Base on $E(\theta)$ minima, the state and consequently the direction of the object magnetization, can be calculated.

Dynamics of the system in high temperature can be discussed in the frame of so-called two-level energetic model [5]. Two magnetic directions of object correspond 

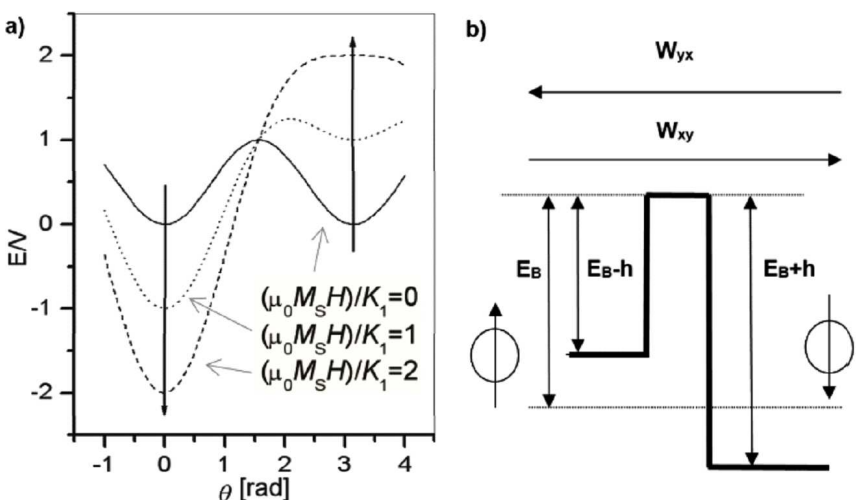

Fig. 2. (a) Examples of $E(\theta)$ dependences based on the Stoner-Wohlfarth model, (b) diagram of the two-level model [6].

to two states, let us say $X$ and $Y$, separated by the blocking energy $E_{B}$ and factor $h$ related to energy interaction between magnetic object and magnetic field. Note that such barrier is asymmetric as shown in Fig. 2b. The frequency of jumps between states is expressed by factors $W_{X Y}\left(\right.$ from $X$ to $Y$ ) and $W_{Y X}$ :

$$
\begin{aligned}
& W_{X Y}=W_{0} \exp \left(\frac{-E_{B}-h}{k_{\mathrm{B}} T}\right), \\
& W_{Y X}=W_{0} \exp \left(\frac{-E_{B} h}{k_{\mathrm{B}} T}\right)
\end{aligned}
$$

Let us assume that total amount of magnetic object is a sum of objects in state $\mathrm{X}$ and Y, i.e. $N=N_{X}+N_{Y}$, then

$$
N_{X}=N \tau W_{Y X}+\left(N_{X 0}-N \tau W_{Y X}\right) \exp \left(\frac{-t}{\tau}\right),
$$

where $\tau=1 /\left(W_{X Y}+W_{Y X}\right)$ and $N_{X 0}=N_{X}(t=0)$.

Note that in the $\mathrm{S}-\mathrm{W}$ model and without external magnetic field, the system has two equivalent minima i.e., at parallel and antiparallel alignment of the particle magnetization to the easy magnetization axis. We propose the model consisting of the non-equivalent minima related to antisymmetric anisotropy constants - different at $\theta=0$ and $\theta=2 \pi$, as shown in Fig. 3 the energy of the system, as a function of $\theta$, can be written as follows:

$$
\begin{aligned}
E_{i} & =K_{1}^{i} \sin \left(\theta_{0}^{i}-\theta\right)^{2}+K_{2}^{i} \sin \left(\frac{\theta_{0}^{i}-\theta}{2}\right)^{2} \\
& -\mu_{i} \mu_{\mathrm{B}} \mu_{0} H
\end{aligned}
$$

where $i$ is the number of the object, $K_{1}^{i}$ is the symmetric part of the anisotropy constant, $K_{2}^{i}$ is the antisymmetric part of the anisotropy constant, $\theta^{i}$ is the angle between the easy magnetization axis and the direction of the external magnetic field, $\mu_{i}$ is the magnetic moment of the object, $\mu_{\mathrm{B}}$ is the Bohr magneton, $\mu$ is the vacuum magnetic permeability, $H$ is the external magnetic field.

Based on the modified $\mathrm{S}-\mathrm{W}$ and the two-level model one may simulate hysteresis loops at different temperatures and for the system containing soft and hard mag-

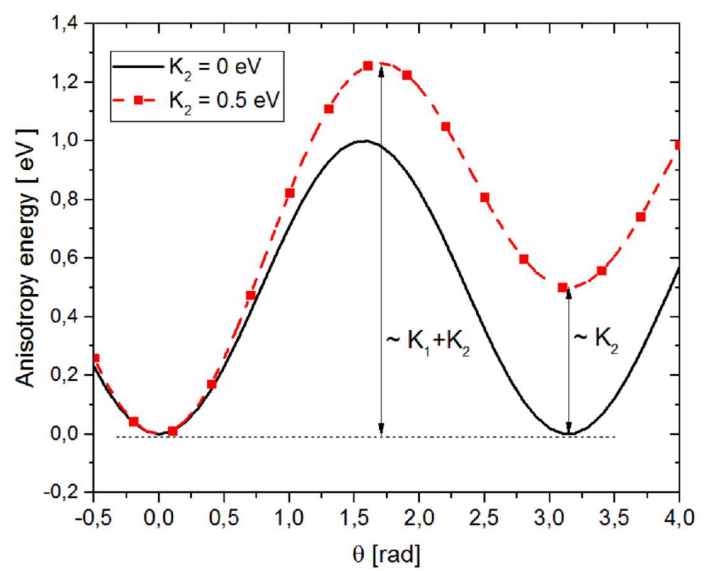

Fig. 3. The anisotropy energy in the modified StonerWohlfarth model, including antisymmetric anisotropy constants.

netic objects. The applied model consists of 5000 magnetic objects with $K_{1}=1 \mathrm{eV}, \mu=10000 \mu_{\mathrm{B}}$ and full range of $\theta$. Moreover, the simulations were carried out for three different temperatures and $K_{2}$ equal to $0,0.3$, and $0.6 \mathrm{eV}$, respectively.

The procedure were carried out in the following steps.

Step 1. Initialize $i$ magnetic objects in random state (random $\theta^{i}$ ).

Step 2. Calculate energy of each object base on the formula (4).

Step 3. Find minima of $E_{i}(\theta)$ and calculate amount of objects in $X$ and $Y$ state based on Eqs. (3). The energy barriers $\left(E_{B}-h\right.$ and $\left.E_{B}+h\right)$ in Eqs. (1) and (2) are determined from the calculated $E_{i}(\theta)$ dependences.

Step 4. Calculate the magnetic moment for each object as $\left\langle\mu_{i}\right\rangle=\mu \mu_{\mathrm{B}}\left(N_{Y} \cos \theta_{Y}^{i}+N_{X} \cos \theta_{X}^{i}\right)$, where $\theta_{Y}^{i}$ and $\theta_{X}^{i}$ are the values of $\theta$ for $Y$ and $X$ state energy minimum of $i$-th object, respectively.

Step 5. Calculate the total magnetic moment $\mu_{\text {tot }}=$ $\sum_{i}\left\langle\mu_{i}\right\rangle$ of the system and update the $H$ value.

Step 6. Repeat the procedure starting from step 2 for full simulation of hysteresis loop at appropriate temperature in $H$ range from $-2.5 \mathrm{~T}$ to $+2.5 \mathrm{~T}$ including 100 points.

\section{Results and discussion}

Figure 4 shows the simulated magnetic hysteresis loops at room temperature for $K_{1}=1 \mathrm{eV}$ and various values of $K_{2}$ equal to $0,0.3$, and $0.6 \mathrm{eV}$. The presented magnetic moment was normalized by taking into account the maximum for the system in magnetic saturation state. In case of $K_{2}=0 \mathrm{eV}$, the hysteresis loop is typical like for single phase non-interacting system that confirms correctness of the applied models. For the values of $K_{2}$ higher than zero, one may observe, let us say, two phase hysteresis shape. Due to the random alignment of the objects 


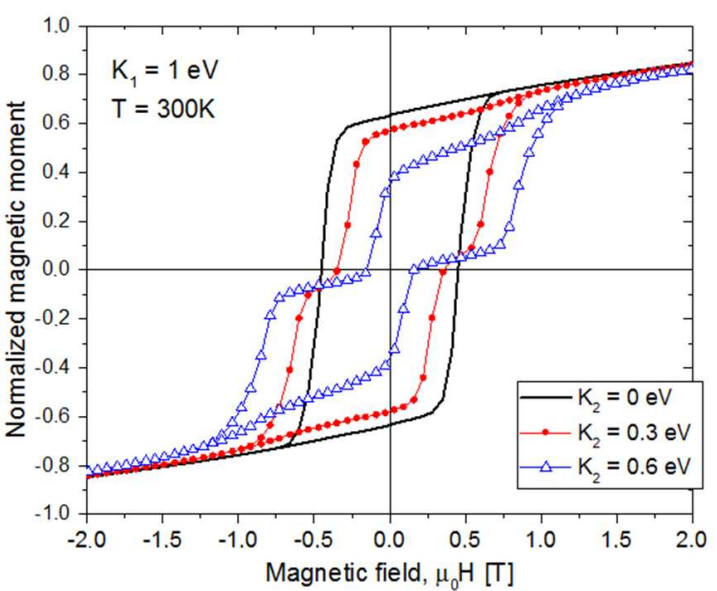

Fig. 4. The simulated hysteresis loops obtained for the one component system with different antisymmetric anisotropy contribution $K_{2}$.

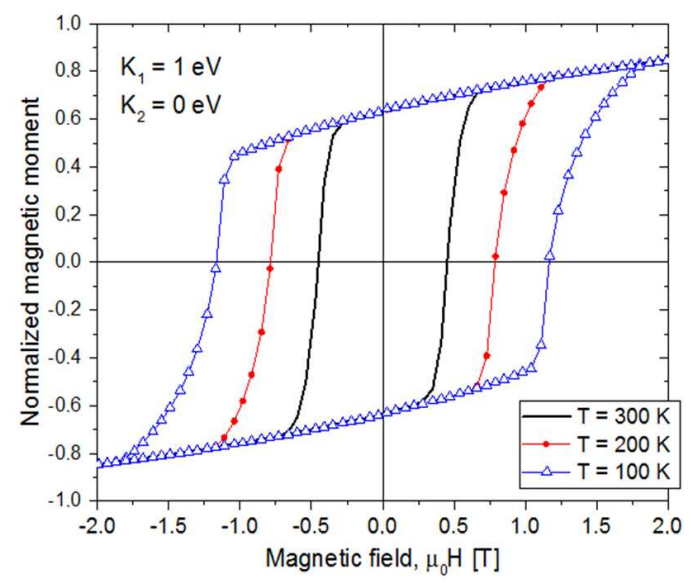

Fig. 5. The simulated hysteresis loops obtained for $K_{2}=0$ at different temperature.

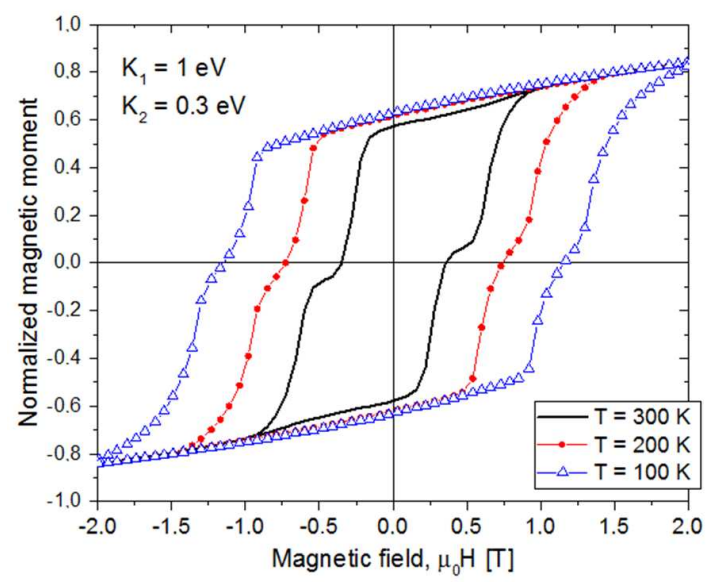

Fig. 6. The simulated hysteresis loops obtained for $K_{2}=0.3$ and different temperature.

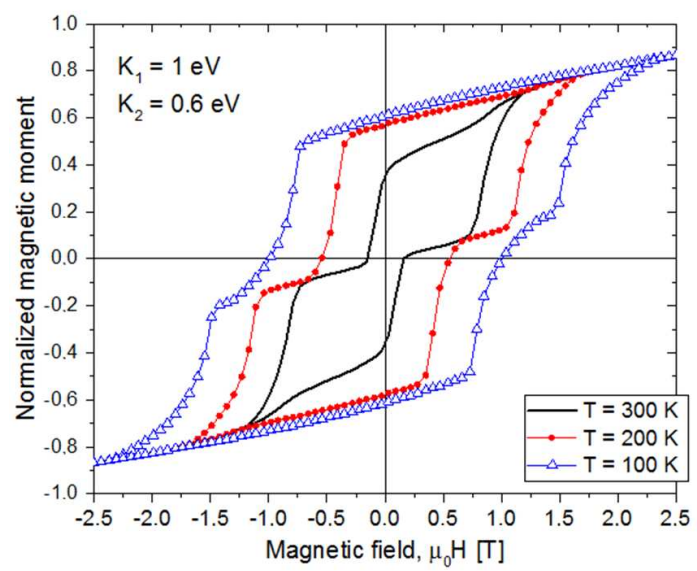

Fig. 7. The simulated hysteresis loops obtained for $K_{2}=0.6$ at different temperature.

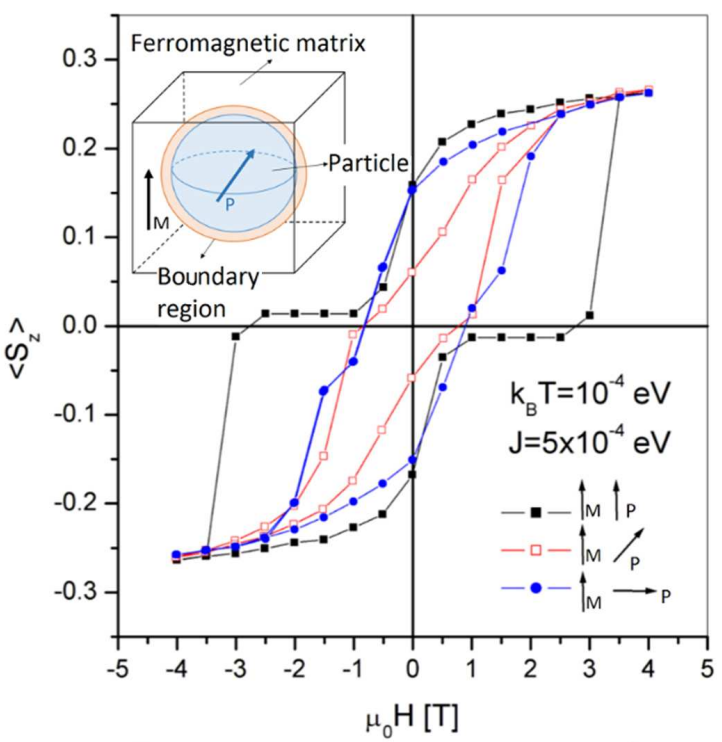

Fig. 8. Average value of spin for the magnetically soft spherical particle embedded into hard magnetic matrix for parallel, $45^{\circ}$, and perpendicular directions of the easy magnetization axes of the particle and the matrix. For details see [7].

(uniform distribution of $\theta^{i}$ ), the steps in the magnetization precession are directly related to the antisymmetric energy barrier caused by the non-zero $K_{2}$ parameter (see Fig. 2). Figures 5 to 7 present impact of temperature on magnetic hysteresis loop obtained for $\mathrm{K}_{2}=0,0.3$, and $0.6 \mathrm{eV}$, respectively. In lower temperature the coercive field increases and relative impact of applied asymmetry decreases. Such effect is expected due to lowering of thermal energy and, in consequence, lowering of frequency of jumps (see formulae (1) and (2)).

It is especially interesting to compare the hysteresis loops obtained in the frame of the proposed model and direct simulations in atomic level. Recently, we have pre- 
sented the Monte Carlo simulations related to a magnetically soft spherical particle embedded into hard magnetic matrix [7], see the inset in Fig. 8. The temperature $k_{\mathrm{B}} T$ equals $10^{-4} \mathrm{eV}$ ( $k_{\mathrm{B}}$ is the Boltzmann constant) and the exchange parameter between the particle and the matrix $J$ equals $5 \times 10^{-4} \mathrm{eV}$. As shown in Fig. 8, the resulting hysteresis loops (accounting parallel and antiparallel alignment of the particle) reveal double phase shapes that are attributed to the particle-matrix exchange coupling. Let us note that in the both cases, i.e. the Monte Carlo simulations and the presented "macroscopic" model, one can observe the double phase hysteresis loops that are originating from single phase of soft magnetic particles and the interactions between them and the ultra-high coercive phases.

The presented comparison confirms that in the case of the system containing interacting soft and ultra-hard magnetic objects, the resulting exchange anisotropy can be simulated by the proposed introduction of the antisymmetric anisotropy constants. Moreover, such double phase hysteresis loops are experimentally observed (see Fig. 1) and they can be understood based on the proposed approach.

\section{Conclusions}

The main conclusions can be summarized as follows:

- The Stoner-Wohlfarth model supplemented by the two-level model can be used in simulations of magnetization processes at higher than zero temperatures.

- In the case of the system containing interacting magnetically soft and ultra-hard objects, the exchange anisotropy can be simulated by the antisymmetric anisotropy constants. Applying of the presented approach results in the occurrence of double magnetic phase hysteresis loops, while in the system only the soft particles can directly contribute to magnetization.

- The correctness of the proposed model was confirmed by the quantitative agreement with the direct Monte Carlo simulations of similar magnetic systems. This means that the direct interactions between soft and ultra-hard magnetic phases can be simulated by the antisymmetric anisotropy constants.

\section{Acknowledgments}

This work was supported by National Science Centre in Poland by the grant no. 2015/19/B/ST8/02636.

\section{References}

[1] K.H.J. Buschow, F.R. de Boer, Physics of Magnetism and Magnetic Materials, Kluwer Academic, New York 2004.

[2] N. Randrianantoandro, A.D. Crisan, O. Crisan, J. Marcin, J. Kovac, J. Hanko, J.M. Grenèche, P. Svec, A. Chrobak, I. Skorvanek, J. Appl. Phys. 108, 093910 (2010).

[3] A. Chrobak, G. Ziółkowski, N. Randrianantoandro, J. Alloys Comp. 583, 48 (2014).

[4] E.C. Stoner, E.P. Wohlfarth, Philos. Trans. R. Soc. A 240, 599 (1948).

[5] G. Haneczok, Migrational Relaxation in Solids, University of Silesia Publ. House, Katowice 2011.

[6] A. Chrobak, Archiv. Mater. Sci. Eng. 58, 80 (2012).

[7] G. Ziółkowski, A. Chrobak, Acta Phys. Pol. A 127, 597 (2015). 